\title{
Beyond HERA: Contributions of specific prefrontal brain areas to long-term memory retrieval
}

\author{
RANDY L. BUCKNER \\ Washington University, St. Louis, Missouri
}

\begin{abstract}
Recent neuroimaging studies have provided a wealth of information about areas within prefrontal cortex involved in long-term memory. These studies prompted a proposal by Tulving and colleagues (Tulving, Kapur, Craik, Habib, \& Houle, 1994) that prefrontal contributions to memory function are related to laterality differences (the hemispheric encoding/retrieval asymmetry model). This review goes beyond a general characterization of prefrontal lobes to a more specific analysis of distinct areas within the prefrontal cortex. Separate prefrontal areas, sometimes within the same hemisphere, are discussed in terms of selective contributions that they might make to memory retrieval. In the end, it is concluded that a framework which tries to understand prefrontal function in terms of specific areas is a useful complement to models, like HERA, which attempt to find unifying principles across multiple areas.
\end{abstract}

The past decade has seen an outpouring of information about the neurobiological basis of human cognition, owing largely to the refinement of methods for imaging active brain areas in healthy, awake subjects as they perform cognitive tasks (Posner \& Raichle, 1994). These methods, often referred to as neuroimaging techniques, provide a window, previously unavailable, into the function of the brain. Memory research has likewise benefitted tremendously from these technologies, with over 50 imaging studies of memory reported to date. Importantly, these studies have allowed researchers to propose and test ideas about brain areas and pathways that might underlie memory function (for examples see Buckner \& Tulving, 1995; Petrides, Alivisatos, Evans, \& Meyer, 1993; Raichle et al., 1994; Schacter, Alpert, Savage, Rauch, \& Albert, 1996). Many of the results are in good agreement with predictions based on earlier methodologies; other results have been entirely unanticipated.

On the basis of unexpected findings about the lateralization of prefrontal activations during memory tasks, Tulving, Kapur, Craik, Moscovitch, and Houle (1994) proposed a model of hemispheric encoding/retrieval asymmetry (HERA). The HERA model drew on data from

I thank Steve Petersen, Julie Fiez, Adina Roskies, and Susan Courtney for helpful comments on various versions of this manuscript and Dan Schacter, Roger Ratcliff, John Gabrieli, Henry Roediger, and an anonymous reviewer for thoughtful comments during the review process. Support was provided by NIH Grant NS32979 and grants from the Charles A. Dana Foundation and the McDonnell Center for Higher Brain Function. The present research was conducted at Washington University School of Medicine, Department of Neurology and Neurological Surgery. Correspondence concerning this article should be sent to R. L. Buckner, MGH-NMR Center, 13th St., Building 149 , Room 2301, Charlestown, MA 02129 (e-mail: buckner@nmr.mgh. harvard.edu). multiple neuroimaging studies conducted across several different laboratories. The main assertions of the HERA model were that (1) right prefrontal cortical areas are more involved in episodic retrieval than are left prefrontal areas, and (2) left prefrontal areas are more involved in semantic retrieval and episodic encoding than are right prefrontal areas. The idea that the prefrontal cortex may be involved in long-term memory function had been previously advocated on the basis of lesion work (Milner, Petrides, \& Smith, 1985; Moscovitch, 1982; Schacter, 1987; Shimamura, Janowsky, \& Squire, 1991), but hemispheric asymmetry in relation to memory function was a completely novel proposal which helped draw attention to the commonalities observed across a wide range of neuroimaging studies.

Recently, the HERA model has been revisited in order to determine how well it has predicted new data (Nyberg, Cabeza, \& Tulving, 1996). The current scorecard, which includes data collected after HERA's initial report (Tulving, Kapur, Craik, et al., 1994), is well in favor of HERA, suggesting that the model reliably captures a general pattern observed across many neuroimaging studies.

However, in spite of the HERA model's ability to predict data on the average, several of its shortcomings need to be addressed. The HERA model's main limitation is that it is minimally constrained in terms of prefrontal anatomy and thus provides only an initial heuristic from which to work. A second limitation is that the model focuses on prefrontal brain areas that differ across stages and types of memory, while it underemphasizes commonalities - which may also be quite important.

In this review, attention will be focused on a finer level of prefrontal analysis in line with our current understanding of prefrontal anatomy. This should not be taken as a step backward. The goal is not to show that the HERA model is inaccurate, but rather to provide a more detailed 
look at which prefrontal areas are involved in memory function, and in what capacities. This is meant as a step forward and as a complement to the HERA model.

Evidence shows that neuroimaging techniques are capable of resolving distinct subdivisions (areas) of the prefrontal cortex. Examination of the prefrontal cortex in terms of specific areas should yield a more complete description of prefrontal contributions to memory. Several examples will be given which show how multiple, functionally distinct prefrontal areas contribute to longterm memory retrieval processes; some of these areas are located in the right prefrontal cortex, while others are located in the left. Neuroimaging studies have revealed distinct prefrontal areas which have selective roles in memory retrieval that are not captured by descriptions based solely on their laterality.

\section{CONCEPTUAL CONSIDERATIONS Multiple Prefrontal Areas and the Ability of Neuroimaging to Detect Them}

Before a description of prefrontal contributions to memory can be made, it is important to discuss what, exactly, the prefrontal cortex is. The prefrontal cortex is a subdivision of the frontal cortex, the most anterior portion of the human brain. Unlike the primary motor cortex and premotor cortex, which are also subdivisions of the frontal cortex, the prefrontal cortex does not have connections to brain areas that directly control movement and thus must act indirectly (through the premotor and motor cortex) to participate in the production of overt behaviors. Anatomically, the prefrontal cortex is defined by the organization of its cortical layers at a microscopic level, its connections to other brain areas such as the thalamus, and physiological properties (GoldmanRakic, 1987).

It has long been accepted that the prefrontal cortex is made up of multiple, functionally distinct areas. Brodmann (1909/1994), using purely anatomical techniques, characterized the human prefrontal cortex and described (with some uncertainty) eight distinct prefrontal areas. The map that he produced, which included these areas and a few more added later, is now widely used as a common reference system. More modern characterizations have further subdivided (and sometimes revised) Brodmann's map in humans (e.g., Petrides \& Pandya, 1994).

Studies of nonhuman primates, which are amenable to more sophisticated anatomical analysis, have revealed numerous distinct areas of the prefrontal cortex with a considerable degree of certainty (Barbas \& Pandya, 1989; Carmichael \& Price, 1994; Preuss \& Goldman-Rakic, 1991). These divisions are far more elaborate than characterizations based on early anatomical approaches using techniques available to Brodmann.

The degree to which the multiple prefrontal areas are interdependent and/or process information in parallel pathways remains unresolved. What is clear is that multiple prefrontal areas have somewhat distinct processing roles-a property that certainly extends to humans. Thus, with regard to the human prefrontal cortex, it is essential to begin a functional characterization with the assumption that multiple areas exist which may perform separable operations (Buckner \& Petersen, 1996).

Given this orientation, the natural question becomes, Are we technically capable of studying the multiple functional subdivisions of human prefrontal cortex? Using traditional methodologies, such as lesion analysis, it is often difficult to divide the prefrontal cortex into functional areas because of the limited availability of focal brain injuries. However, such characterization is beginning and is likely to make important contributions (e.g., Fiez, Damasio, \& Tranel, 1995; Swick \& Knight, in press).

Neuroimaging methods, however, serve as a ready tool for characterizing distinct prefrontal areas. These methods permit the observation of local changes in metabolism that are correlated with focused changes in neural activity (DeYoe, Bandettini, Neitz, Miller, \& Winans, 1994; Raichle, 1987). Moreover, there are data that can be used to determine the current ability of neuroimaging to localize and resolve activations within the prefrontal cortex-localization referring to the accuracy with which an activation can be placed within the prefrontal cortex, and resolution referring to the distance by which two areas of activation must be separated before they can be identified as more than one area. Localization and resolution are similar in that they both enable the accurate description of an activation. However, multiple areas of activation can be indistinguishable (poor resolution) but nonetheless consistently localized to the same large area (reliable localization). Similarly, multiple activated areas can consistently appear as separate activations (good resolution), but their locations can vary from one subject group to the next (unreliable localization). It is thus important to address localization and resolution separately.

The ability of neuroimaging to reliably localize prefrontal brain areas is demonstrated in studies of the same behavioral paradigm across independent groups of subjects imaged on separate occasions. Figure 1 A shows such an example, in which a left prefrontal activation was detected across three independent groups of subjects performing the same verbal memory retrieval task (Buckner, Petersen, Ojemann, et al., 1995). The anatomical region covered by the activations is relatively small and suggests about a $10-\mathrm{mm}$ range in the ability to localize activations in groups of subjects. A slightly better result was obtained with a right prefrontal activation in the same groups of subjects performing a different verbal retrieval task (see Buckner \& Petersen, 1996). Examination of male and female subjects separately also showed reliable localization of prefrontal activations within the millimeter range (Buckner, Raichle, \& Petersen, 1995). These findings clearly demonstrate that current neuroimaging techniques are highly reliable at detecting prefrontal activations.

Equally essential is the ability of neuroimaging to resolve activations originating from distinct prefrontal areas as separate. ${ }^{1}$ The first evidence that neuroimaging 

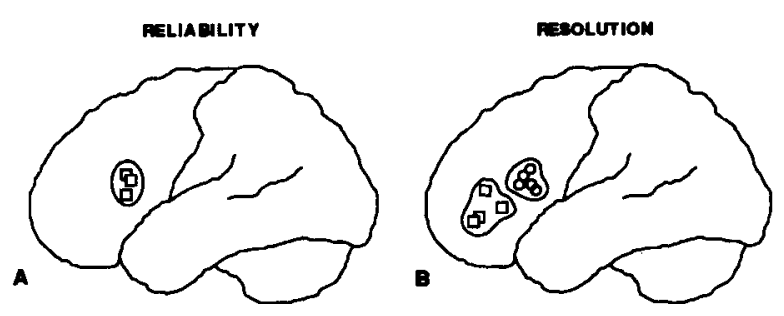

Figure 1. Two lateral views of the left hemisphere illustrate the reliability and resolution of PET neuroimaging to detect activations within human prefrontal cortex (see note 1). (A) Three activations are plotted that were obtained from independent groups of subjects performing the same task (squares). As can be seen, the activations fall in nearly the same location across groups, suggesting that the technique is highly reliable (Buckner, Petersen, et al., 1995). (B) Two clusters of activations are plotted from independent subject groups performing two different tasks. The circles represent an area activated during both tasks; the squares represent an area that is activated during only one of the tasks. Demonstration that these two areas can be reliably separated by manipulating behavioral tasks resolves the two areas as being functionally independent (Buckner, Raichle, et al., 1995). Figure adapted, with permission, from "What Does Neuroimaging Tell Us About the Role of Prefrontal Cortex in Memory Retrieval?" by R. L. Buckner and S. E. Petersen, 1996, Seminars in the Neurosciences, 8, pp. 47-55. Copyright 1996 by Academic Press.

can resolve multiple prefrontal areas comes from a review of the literature. There have been reports of activations that span nearly the entire volume of prefrontal cortex $-\mathrm{a}$ large region that, as discussed earlier, is known to contain multiple anatomically distinct areas. However, there are many sources of variance that might erroneously produce the appearance of multiple prefrontal areas' being activated (e.g., anatomic variability, different localization procedures used across laboratories, inclusion of data that contain Type I errors).

For this reason, it is important to determine a more conservative estimate of neuroimaging's ability to distinguish among prefrontal areas (in this instance, PET neuroimaging). This can be done by directly comparing data across behavioral tasks and showing that one prefrontal area is reliably activated by one set of tasks, whereas a nearby area is reliably activated by a different set of tasks. ${ }^{2}$ Several recently published analyses using this kind of approach suggest a current upper limit to the resolution of neuroimaging at about $16 \mathrm{~mm}$ (Buckner, Raichle, \& Petersen, 1995; Petrides, Alvisatos, Evans, \& Meyer, 1993) (Figure 2B), which corresponds roughly to the difference between the approximate locations of Brodmann areas 10 and 45 , or areas 44 and 47.

If we extrapolate from nonhuman primate work, it seems likely that the currently available resolution is quite adequate for observing the first level of functional subdivisions within the prefrontal cortex. As research progresses, it will probably be necessary to observe physiological function at a much finer resolution, and neuroimaging may not be the tool for this next level of analysis. It is also important to acknowledge that changes in patterns of neuronal firing that occur below this resolution (perhaps even at the millimeter level) will go undetected, because the visualized pattern of activity is blurred over a larger spatial extent. Nonetheless, neuroimaging clearly provides a method for localizing and resolving human prefrontal activations to a level previously unavailable, and it can be exploited to gain insights into prefrontal functional anatomy.

\section{PREFRONTAL PATHWAYS USED DURING MEMORY RETRIEVAL}

Given that the prefrontal cortex is made up of multiple areas and that current neuroimaging techniques can detect activations within these areas, it can be shown that distinct prefrontal areas are activated selectively during different kinds of memory retrieval. Rather than start with a discussion of a large number of retrieval tasks, I will begin by describing findings from three separate sets of task pairs.

These task pairs can serve to illustrate the main point of this review: that functionally distinct (and sometimes multiple) prefrontal areas are used during memory tasksduring both episodic and semantic retrieval tasks. Because neuroimaging data are noisy and the literature likely contains reports of spurious activations, the three task pairs have been selected so as to include only findings replicated across independent groups of subjects performing the same (or very similar) tasks (see Table 1). I am thus highly confident that these task pairs can be used to dissociate multiple prefrontal brain areas. The descriptions of the processes that the areas support, on the other hand, will likely be refined as more data are collected. Two of the prefrontal areas that have been identified will be discussed in more detail, in order to illustrate their generality.

\section{Comparison of Two Different Verbal \\ Semantic Retrieval Tasks}

Semantic retrieval refers to accessing facts and general information learned over the course of one's lifetime, without recollection of where or when the information was learned (Tulving, 1983). The most common example of semantic retrieval, and the one most studied with the use of neuroimaging, is word retrieval. Two semantic word retrieval tasks will be contrasted here: one involving the retrieval of words based on letter cues and the other involving the retrieval of words based on word meaning.

The first task is the stem-completion task. In this task, subjects are given a series of three-letter word beginnings (e.g., "COU") and asked to complete each one to form a word (e.g., "couple" or "cousin"). Clearly, many component processes must be completed for one to succeed at this task. Subjects must visually process the letters, extract their identity, retrieve a word based on the letters, and verbally output that word. When subjects are imaged as they perform this task, it can be seen that 


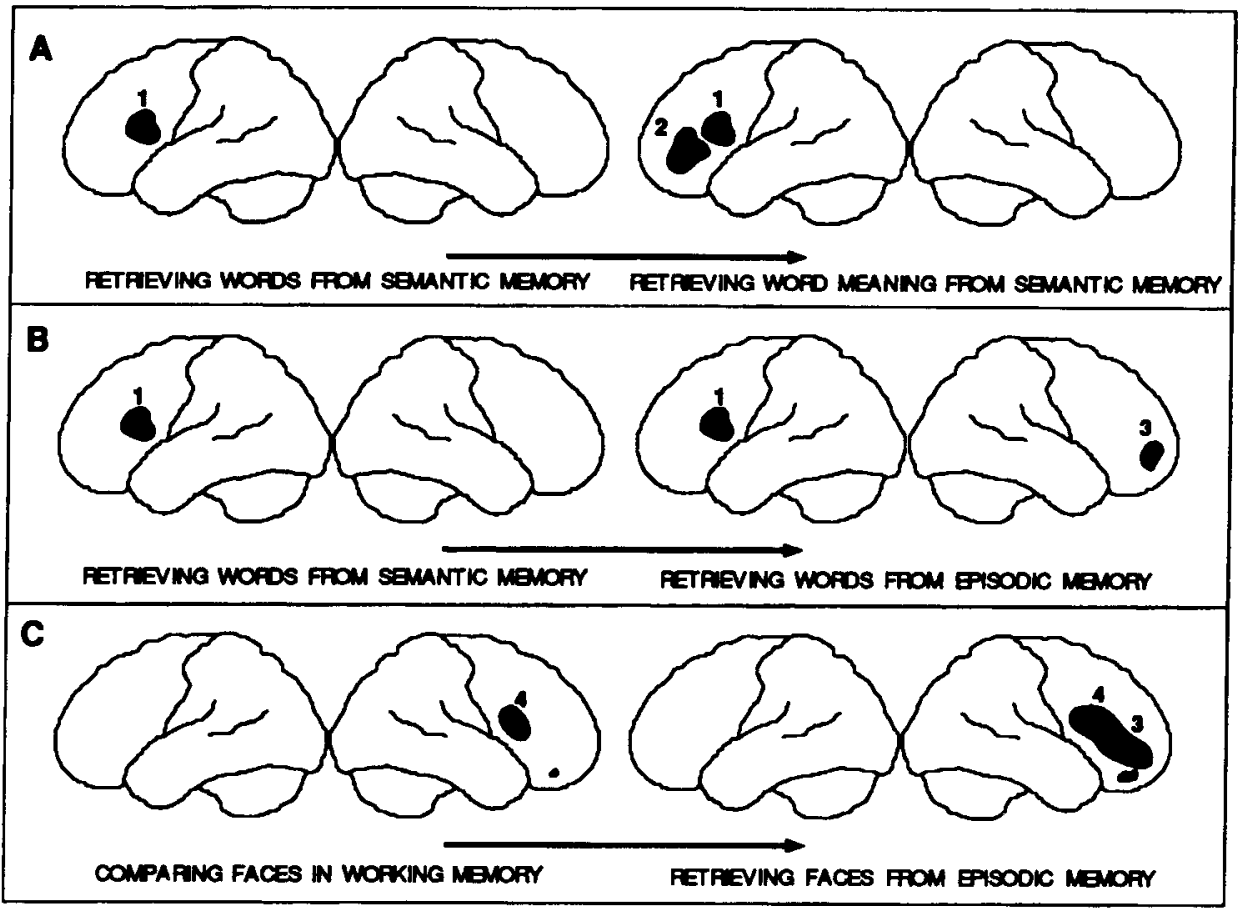

Figure 2. Three sets of tasks are depicted to illustrate memory-related activation of separate prefrontal areas. Each pair of hemispheres shows left and right lateralized activations from a single task image, with activations indicated by shading. (A) Activations across two tasks relying on verbal semantic retrieval are illustrated. Two separate areas in the left prefrontal cortex (labeled 1 and 2 ) are activated differentially across the two tasks (see text). (B) A comparison between a task relying on verbal semantic retrieval and one relying on verbal episodic retrieval is shown. This comparison reveals a specific right anterior prefrontal area activated only during episodic retrieval (labeled 3) (see text). (C) Two tasks are compared that utilize nonverbal (face) information. Remarkably, when the task involves episodic retrieval of faces, the same right anterior prefrontal area is activated as in verbal episodic retrieval (labeled 3). However, in the case of face retrieval, this area is activated in addition to a more posterior right-lateralized area (labeled 4) that is not present in any of the verbal tasks.

many of their brain areas are activated (compared with a low-level reference control task, in which the subjects stare at a fixation point). The activation of several of these brain areas is attributable to the visual and speech demands of the task; these activated areas include striate and extrastriate cortex (visual cortex), premotor and motor cortex, medial cerebellum, and the supplementary motor area. In addition to these areas, anterior cingulate, rightlateral cerebellum, and left inferior prefrontal cortex are also activated.

Of interest here is the left inferior prefrontal area, depicted in the left panel of Figure 2A (labeled 1). This area, localized at or near Brodmann areas 44 and/or 45, has been activated reliably during stem completion (Buckner, Petersen, et al., 1995). ${ }^{3}$ It appears to perform, in some capacity, operations related to semantic word retrieval. ${ }^{4}$ The evidence for this is that it is activated across a wide range of other tasks that involve verbal semantic retrieval (as will be discussed later). Moreover, it does not appear to be activated during tasks that involve simple speech output which do not require subjects to access a new word from memory (see Buckner \& Petersen, 1996; Buckner \& Tulving, 1995; or Raichle et al., 1994, for discussion).
Examination of the second semantic retrieval task, in which subjects must retrieve words on the basis of word meaning, reveals a quite different pattern of left prefrontal activation (Petersen, Fox, Posner, Mintun, \& Raichle, 1988, 1989; Raichle et al., 1994). Instead of the activation's being confined to a relatively small area of the left prefrontal cortex, the activated region now extends anteriorly and ventrally into Brodmann areas 10 and/or 47 (but still includes the area activated by stem completion). The right panel of Figure 2A shows this more diffuse activation (areas labeled 1 and 2). Of central importance here is that this pattern of more widespread activation does not reflect noise or random variables; the same pattern has been shown to occur independently across two separate groups of subjects (Buckner, Raichle, \& Petersen, 1995). A reasonable (but still tentative) explanation is that, during the retrieval of word meaning, a second level of more anterior left prefrontal areas is recruited in addition to the left inferior prefrontal area. Support for this explanation comes from work of Kapur et al. (1994) and Demb et al. (1995), who have also found activation of these more anterior areas during tasks involving the semantic retrieval of word meaning (but see Klein, Milner, Zatorre, Meyer, \& Evans, 1995). 
Table 1

Sources of Data Providing the Three Examples of Dissociations in Prefrontal Cortex Drawn in Figure 2

\begin{tabular}{|c|c|}
\hline Initial Source of Data & Replication(s) \\
\hline $\begin{array}{l}\text { Figure 2A: left panel } \\
\text { Buckner, Petersen, et al. (1995, Exp. 1) }\end{array}$ & Buckner, Petersen, et al. (1995, Exp. 2, Exp. 3)* \\
\hline $\begin{array}{l}\text { Figure 2A: right panel } \\
\text { Petersen et al. (1988); Petersen et al. (1989) }\end{array}$ & Raichle et al. (1994)* \\
\hline $\begin{array}{l}\text { Figure 2B: left panel } \\
\text { Buckner, Petersen, et al. (1995, Exp. 1) }\end{array}$ & Buckner, Petersen, et al. (1995, Exp. 2, Exp. 3) \\
\hline $\begin{array}{l}\text { Figure 2B: right panel } \\
\text { Squire et al. (1992) }\end{array}$ & Buckner, Petersen, et al. (1995, Exp. 2, Exp. 3) \\
\hline $\begin{array}{l}\text { Figure 2C: left panel } \\
\text { Haxby et al. (1994) }\end{array}$ & Grady et al. (1994, old subjects $)^{\dagger}$ \\
\hline $\begin{array}{l}\text { Figure 2C: right panel } \\
\text { Haxby et al. (1996) }\end{array}$ & Grady et al. (1995, old subjects) \\
\hline
\end{tabular}

Taken together, these two semantic retrieval tasks reveal something about the organization of the left prefrontal cortex. A left inferior area appears to be generally recruited when subjects must access word information (area 1, Figure 2A). A second area (area 2, Figure 2A), appears to be additionally recruited when the semantic retrieval requires access to word meaning. The interpretations of the functional basis for these activations are, of course, tentative, because such a small number of instances exist from which to generalize. Regardless, the dissociation between the left inferior prefrontal area and the more anterior left prefrontal areas is robust and is not predicted by the HERA model. Any new models of prefrontal function should take this dissociation into account (Buckner, Raichle, et al., 1995).

\section{Comparison of Verbal Semantic and Episodic Retrieval}

Episodic retrieval refers to the recollection of a specific event and the context associated with that event. This differs from semantic retrieval in that, for episodic retrieval, information must be accessed that contains the individual perspective of the rememberer and information about where and when the event occurred (Tulving, 1983). A reasonable question to ask is, What are the similarities and differences among brain areas activated during semantic and episodic memory retrieval tasks?

This question was addressed in a series of studies by Squire et al. (1992) and Buckner, Petersen, et al. (1995). The task studied was a variant of the stem-completion task that relied on episodic memory (often called stemcued recall). For this task, subjects studied a list of words (e.g., "courage"). Then, after a delay period, they were shown the beginnings of the words (e.g., "COU") and asked to recall the study words that began with the letters. This differs from the stem-completion task discussed earlier, in that subjects are asked to specifically recall words presented during a unique study episode rather than to produce any word beginning with the three letters.
The retrieval portion of this episodic version of the stem-completion task activated the same brain areas that the semantic version of the task did. These included anterior cingulate, right-lateral cerebellum, and left inferior prefrontal cortex as well as the other brain areas discussed earlier. This answers one part of the question posed earlier: overlapping brain areas are activated during semantic and episodic retrieval. However, in addition to these common areas, a new area in the right anterior prefrontal cortex (Buckner, Petersen, et al., 1995), located at or near Brodmann area 10, was activated (Figure 2B). ${ }^{5}$ This right anterior prefrontal area demonstrates selective activation in the episodic version of the stemcompletion task and is not observed during the exclusively semantic version of the task.

If one considers these data (Figure 2B) and compares them with the data discussed in the previous section (Figure 2A), a pattern emerges. An area in the left inferior prefrontal cortex (area 1, Figure 2) appears to be generally recruited during all the tasks requiring retrieval of verbal information, including both semantic and episodic retrieval tasks. This area may support access to, or representation of, verbal information-a demand that is common to all the retrieval tasks illustrated in Figures $2 \mathrm{~A}$ and $2 \mathrm{~B} .{ }^{6}$ Additional support for this possibility is shown in Figure 3A, in which activations are plotted across a wide range of tasks requiring retrieval of verbal information. This figure shows a remarkable convergence around this distinct area in the left inferior prefrontal cortex.

Even more provocative is the observation that this left inferior prefrontal area is often not activated in isolation. Multiple, possibly higher level, prefrontal brain areas are additionally activated, depending on the specific task demands. In the task involving semantic retrieval of word meaning, the left inferior area was activated in addition to more anterior left prefrontal areas. In the episodic retrieval task just discussed, the left inferior area was activated along with a right anterior prefrontal area. 


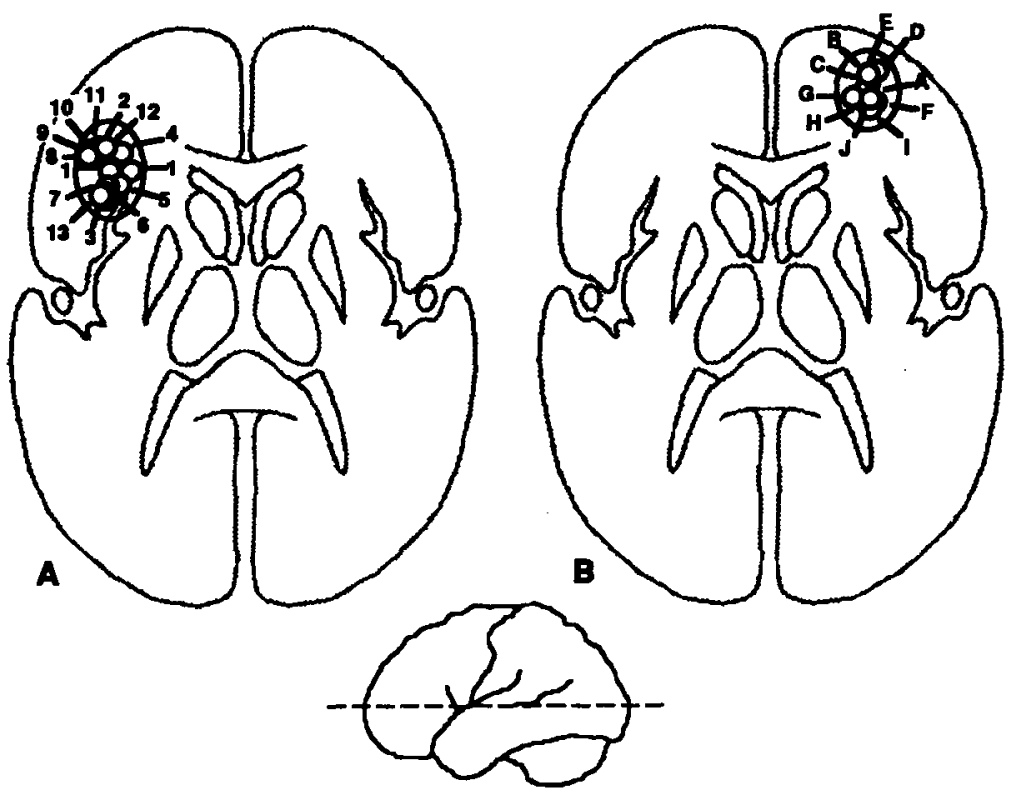

Figure 3. Data from a number of PET studies are plotted to show that highly localized areas of the prefrontal cortex are reliably activated during tasks involving common memory demands. Both sections represent horizontal planes through the brain at $12 \mathrm{~mm}$ above the $\mathrm{AC}-\mathrm{PC}$ line based on Talairach and Tournoux (1988) (the approximate location is depicted in the lateral view of the brain). Activations were included if they fell within $1 \mathrm{~cm}$ of this horizontal plane. Panel A: Verbal memory retrieval. A left inferior area is activated across a wide range of tasks involving semantic (and sometimes episodic) retrieval of verbal information: (1) Petersen, Fox, Posner, Mintun, and Raichle (1988); (2) Frith, Friston, Liddle, and Frackowiak (1991); (3) Wise et al. (1991); (4) Kapur et al. (1994); (5-7) Buckner, Petersen, et al. (1995); (8) Raichle et al. (1994); (9-11) Klein, Milner, Zatorre, Meyer, and Evans (1995); (12-13) Martin, Haxby, Lalonde, Wiggs, and Ungerleider (1995). Panel B: Episodic memory retrieval. A distinct right anterior prefrontal area is activated by many tasks that require episodic retrieval, including tasks involving both verbal and nonverbal information: (A) Squire et al. (1992); (B-C) Buckner, Petersen, et al. (1995); (D) Haxby et al. (1996); (E) Grady et al. (1995); (F) Tulving, Kapur, Markowitsch, et al. (1994); (G-H) Buckner, Raichle, et al. (1995); (I) Andreasen et al. (1995); and (J) Schacter et al. (1996).

The HERA model, by illuminating only differences between episodic and semantic retrieval, draws attention away from the fact that this left inferior prefrontal area is active during both episodic and semantic verbal retrieval. This occurs because the data can be discussed and interpreted in one of two ways, both of which are correct (Figure 4). One way to examine brain areas active during semantic and episodic retrieval tasks is to compare the tasks with a low-level control task that does not make any memory demands. Such comparisons reveal all the brain areas active during the tasks - those which overlap as well as those which are unique to the two tasks. Alternatively, if one wanted to isolate brain areas specific to episodic retrieval, one might compare it with a similar task that required only semantic retrieval. This approach would isolate areas selective for episodic retrieval but would not necessarily reveal all the areas activated by the episodic retrieval task. Areas shared in common by both the semantic and the episodic retrieval tasks would be missed.
There are tradeoffs between the two kinds of comparisons. Well-controlled comparisons serve to better isolate cognitive processes and, presumably, isolate brain areas differentially involved in those processes. Such comparisons, however, potentially miss important brain areas being activated by the task, simply because the reference control task is also activating those brain areas. Comparisons involving low-level reference tasks identify more completely the brain pathways activated but are difficult to interpret because they are underconstrained.

Both of these tradeoffs are illustrated in Figure 4. A distributed pathway including left inferior prefrontal cortex is similarly activated during both semantic and episodic versions of the stem-completion task. As already discussed, a right anterior prefrontal area is additionally activated during the episodic version. It is quite possible that the role of the right anterior prefrontal area cannot be understood independently of the other brain areas that are concurrently being activated. If there had 


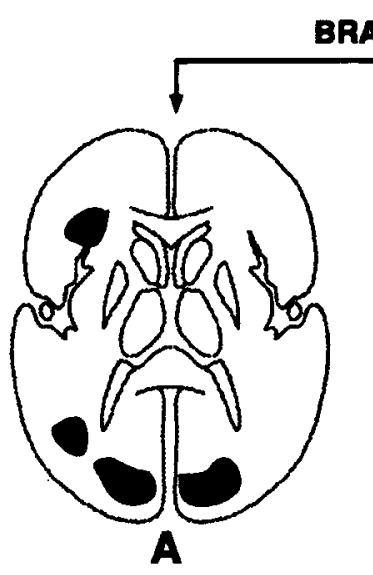

Semantic Potrieval

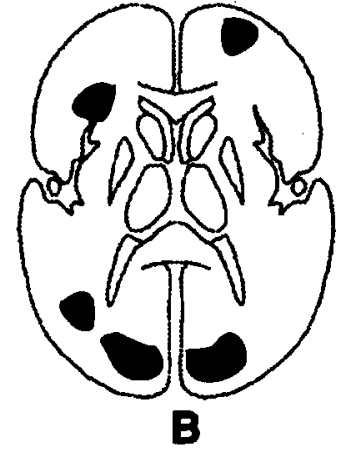

Episodic Rotrieval

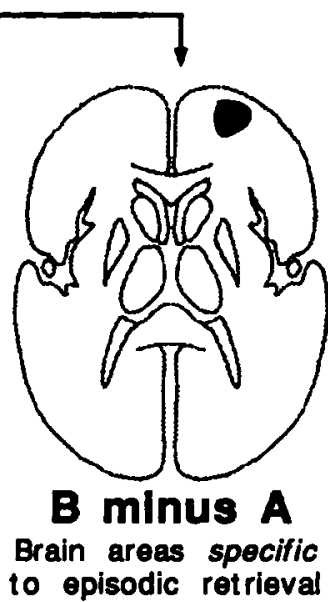

L

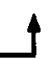

ALL ACTIVE BRAIN AREAS

\begin{abstract}
Figure 4. Representative activations are displayed for two different memory tasks (from Buckner, Petersen, et al., 1995), one relying on semantic retrieval (left section, labeled A) and one relying on episodic retrieval (middle section, labeled $B$ ). The activations displayed in these two sections are determined in relation to a low-level control task which contains minimal memory demands. Making such a broad comparison shows that many cortical brain areas are activated because of the many demands of the memory retrieval tasks, including areas activated in common by the two memory tasks as well as those differentially activated. If, on the other hand, the two tasks are compared directly, then only the brain areas differentially activated across the two kinds of memory tasks are visible (right section, labeled B minus A). The latter kind of comparison allows one to observe brain areas specialized for the processes that differ between the two tasks. However, the complete pattern of activation is only appreciated by observing both kinds of comparisons.
\end{abstract}

been no comparison that involved a low-level control task, only activation of the right anterior prefrontal cortex would have been detected, and the other, potentially important, areas in the pathway would not have been observed. If, on the other hand, the episodic version of the stem-completion task had been compared exclusively with the low-level control task, a different problem would have occurred. Areas within the complex activation pattern specifically related to episodic retrieval would not have been revealed. Ideally, researchers should try to examine as many relevant comparisons as possible in order to understand their data fully. ${ }^{7}$

Many data in the literature are presented using only one kind of comparison, including those predominantly relied on by Tulving and colleagues in their recent explication of the HERA model (Nyberg et al., 1996). For the data that they highlight, almost all of the episodic retrieval tasks were compared with semantic retrieval tasks. It is likely that, for many of the episodic retrieval tasks included in the HERA model - especially those relying on verbal information - other brain areas, including the left inferior prefrontal cortex, were activated and would have been detected if the episodic retrieval tasks had been compared with tasks that did not involve the manipulation of words from semantic memory (see also Petrides, Alivisatos, \& Evans, 1995, for a similar observation).

\section{Comparison of a Working Memory for Faces Versus Episodic Retrieval of Faces}

The tasks thus far discussed have relied on retrieval of verbal information and have revealed a common left inferior prefrontal cortex activation. The task pair discussed here requires processing of pictorial (face) information, and the PET images obtained during their performance demonstrate a different pattern of prefrontal activation.

The first task involves simply deciding which of two faces matches a target face. To perform this task, subjects likely process the visual forms that they are presented, recognize that they are faces, and then make a comparison of the faces in working memory, selectively attending to the features that make the faces distinct. Images of brain activation for this task, as compared with a sensorimotor control task (no faces were present in the control task), show many visual areas in the occipital cortex as well as an area in the right inferior prefrontal cortex (Grady et al., 1994; Haxby et al., 1994; see also Courtney, Ungerleider, Keil, \& Haxby, in press). This rightinferior prefrontal area falls at or near Brodmann areas 45 and/or 47 (area 4, Figure 2C, left panel).

Contrasting this area of right-inferior cortex activation with the verbal memory retrieval tasks discussed earlier demonstrates another interesting distinction among prefrontal areas. In this case, the distinction is between ver- 
bal and pictorial information. The verbal tasks discussed previously (including both semantic and episodic retrieval) activated a left inferior prefrontal area (Figures $2 \mathrm{~A}$ and $2 \mathrm{~B}$ ), whereas the current task, requiring the processing of pictorial information, activated a right inferior prefrontal area (Figure 2C, left panel).

When the face-matching task was changed to require subjects to recognize the faces on the basis of information within episodic memory, a right anterior prefrontal area was observed in addition to the more posterior right inferior area activated by face matching (Grady et al., 1995; Haxby et al., 1996). Remarkably, this right anterior prefrontal area is in a location nearly identical to the area activated by the verbal episodic memory retrieval task (area 3, Figure 3C). These findings suggest two important points. First, they demonstrate that the right anterior prefrontal area is distinct from the more posterior right inferior prefrontal area recruited during both face matching and episodic retrieval of faces. Second, they show that the right anterior prefrontal area is selectively recruited during episodic retrieval regardless of the kind of information being retrieved.

Figure 3B explores this last point more fully by showing activation of this right anterior prefrontal area across 10 episodic retrieval tasks. These episodic retrieval tasks include paired-associate recall of words (e.g., Andreasen et al., 1995; Buckner, Raichle, Miezin, \& Petersen, 1995), paired-associate recall of pictures (e.g., Buckner, Raichle, et al., 1995), recognition of words (e.g., Tulving, Kapur, Markowitsch, et al., 1994), and recognition of faces (e.g., Grady et al., 1995; Haxby et al., 1996). The retrieval cues have been presented aurally (e.g., Buckner, Raichle, et al., 1995; Tulving, Kapur, Markowitsch, et al., 1994) and visually (e.g., Buckner, Petersen, et al., 1995; Grady et al., 1995; Haxby et al., 1996), and the study modality has been systematically manipulated (e.g., Buckner, Petersen, et al., 1995). Across all of these surface changes, the same area has been activated during episodic retrieval. Most importantly, the data presented suggest that the common activation does not occur across a distributed set of right prefrontal areas. Rather, the common activation during episodic retrieval is highly localized, falling at or near Brodmann area 10.

\section{CONCLUSIONS}

This review has demonstrated that neuroimaging enables the observation of activation within specific areas of the prefrontal cortex. The analysis of neuroimaging data with the goal of isolating contributions of specific prefrontal brain areas to memory has highlighted a number of findings that would otherwise be missed within a framework that treats prefrontal cortex as a single entity - such as that presented by the HERA model.

By no means is this meant to suggest that a model like HERA, which attempts to find a unitary function across prefrontal areas, cannot be valid. As Moscovitch (1989) points out in relation to neuropsychological data, "there is something romantic in trying to find unity or at least a family resemblance among the variety of memory deficits after frontal system damage" (p. 157). This idea is echoed in work by Goldman-Rakic (1987), who has postulated, on the basis of nonhuman primate work, that "each subdivision of prefrontal cortex performs a similar operation and differences between areas lie mainly in the nature of information upon which the operation is performed" (pp. 138-139).

However, notions of unitary function within prefrontal cortex (or lobes within prefrontal cortex) should be entertained with moderation. Perhaps there are unifying principles that relate function across prefrontal areas; perhaps groups of areas share similar properties that make them distinct from other sets of areas within the prefrontal cortex. Regardless, in relation to memory, the research goal is the same--to attempt to determine the processing roles of specific brain areas and how they interact with other brain areas. If there are similarities across prefrontal areas, either in the kind of process they perform, or in the kind of information upon which they operate, patterns will emerge that suggest these commonalities. If, on the other hand, the prefrontal cortex is only treated as a single entity, the possibility of understanding the distinct roles of the individual areas is lost from the onset.

\section{REFERENCES}

andreasen, N. C., O'Leary, D. S., Arndt, S., Cizadlo, T., Hurtig, R., Rezai, K., Watkins, G. L., Boles Ponto, L. L., \& Hichwa, R. D (1995). Short-term and long-term verbal memory: A positron emission tomography study. Proceedings of the National Academy of Science, 92, 5111-5115.

Barbas, H., \& Pandya, D. N. (1989). Architecture and intrinsic connections of the prefrontal cortex in the rhesus monkey. Journal of Comparative Neurology, 286, 353-375.

BrodmanN, K. (1994). Localisation in the cerebral cortex ( $\mathrm{L}$. J. Garey, Trans.) London: Smith-Gordon. (Original work published 1909)

Buckner, R. L., \& Petersen, S. E. (1996). What does neuroimaging tell us about the role of prefrontal cortex in memory retrieval? Seminars in the Neurosciences, 8, 47-55.

Buckner, R. L., Petersen, S. E., Ojemann, J. G., Miezin, F. M. Squire, L. R., \& RaICHLE, M. E. (1995). Functional anatomical studies of explicit and implicit memory retrieval tasks. Journal of Neuroscience, 15, 12-29.

Buckner, R. L., Raichle, M. E., Miezin, F. M., \& Petersen, S. E. (1995). PET studies of the recall of pictures and words from memory. Society for Neuroscience Abstracts, 21, 1441.

BuckNer, R. L., Raichle, M. E., \& Petersen, S. E. (1995). Activation of human prefrontal cortex across different speech production tasks and gender groups. Journal of Neurophysiology, 74, 2163-2173.

BuCKNER, R. L., \& TulviNG, E. (1995). Neuroimaging studies of memory: Theory and recent PET results. In F. Boller \& J. Grafman (Eds.), Handbook of neuropsychology (Vol. 10, pp. 439-466). Amsterdam: Elsevier.

Carmichael, S. T., \& Price, J. L. (1994). Architectonic subdivision of the orbital and medial prefrontal cortex in the macaque monkey. Journal of Comparative Neurology, 346, 366-402.

Courtney, S. M., Ungerleider, L. G., Keil, K., \& Haxby, J. V. (in press). Object and spatial visual working memory activate separate neural systems in human cortex. Cerebral Cortex.

Demb, J. B., Desmond, J. E., Wagner, A. D.. Vaidya, C. J., Glover, G. H., \& GabrieLI, J. D. E. (1995). Semantic encoding and retrieval 
in the left inferior prefrontal cortex: A functional MRI study of task difficulty and process specificity. Journal of Neuroscience, 15, $5870-5878$.

DeYoe, E. A., Bandettini, P., Neitz, J., Miller, D., \& Winans, P. (1994). Functional magnetic resonance imaging (fMRI) of the human brain. Journal of Neuroscience Methods, 54, 171-187.

Fiez, J. A., Damasio, H., \& Tranel, D. (1995). The left frontal operculam in phonological processing: Converging evidence from PET and the lesion method. Society for Neuroscience Abstracts, 21, 1763

Fiez, J. A., Raife, E. A., Balota, D., Schwarz, J, P., Raichle, M. E., \& Petersen, S. E. (1996). A positron emission tomography study of the short-term maintenance of verbal information. Journal of Neuroscience, 16, 808-822.

Fletcher, P. C., Frith, C. D., Grasby, P. M., Shallice, T., FrackOWIAK, R. S. J., \& Dolan, R. J. (1995). Brain systems for encoding and retrieval of auditory-verbal memory: An in vivo study in humans. Brain, 118, 401-416.

Frith, C. D., Friston, K. J., Liddle, P. F., \& Frackowiak, R. S. J (1991). Willed action and the prefrontal cortex in man: A study with PET. Proceedings of the Royal Society of London: Series B, 244, $241-246$

FusteR, J. M. (1989). The prefrontal cortex. New York: Raven Press. Goldman-RaKIC, P. S. (1987). Circuitry of primate prefrontal cortex and regulation of behavior by representational memory In F. Plum \& V. Mountcastle (Eds.), The handbook of physiology: Section I. The nervous system: Vol. V. Higher functions of the brain: Pt. 1 (pp. 373-417). Bethesda, MD: American Physiological Society.

Grady, C. L., Maisog, J. M., Horwitz, B., Ungerleider, L. G., MeNTis, M. J., Salerno, J. A., Pietrini, P., Wagner, E., \& Haxby, J. V. (1994). Age-related changes in cortical blood flow activation during visual processing of faces and locations. Journal of Neuroscience, 14, 1450-1462.

Grady, C. L., McIntosh, A. R., Horwitz, B., Maisog, J. M., UngerLeider, L. G., Mentis, M. J., Pietrini, P., Schapiro, M. B., \& HAXBY, J. V. (1995, July 14). Age-related reductions in human recognition memory due to impaired encoding. Science, 269, 218-221.

Haxby, J. V., Horwitz, B., Ungerleider, L. G., Maisog, J. M. Pietrini, P., \& Grady, C. L. (1994). The functional organization of human extrastriate cortex: A PET-rCBF study of selective attention to faces and locations. Journal of Neuroscience, 14, 6336-6353.

HaXby, J. V., Ungerleider, L. G., Horwitz, B., Maisog, J. M. RAPOPORT, S. L., \& GRADY, C. L. (1996). Face encoding and recognition in the human brain. Proceedings of the National Academy of Science, 93, 922-927.

Kapur, S., Craik, F. I. M., Tulving, E., Wilson, A. A., Houle, S., \& Brown, G. M. (1994). Neuroanatomical correlates of encoding in episodic memory: Levels of processing effect. Proceedings of the National Academy of Science, 91, 2008-2011.

Klein, D., Milner, B., Zatorre, R. J., Meyer, E., \& Evans, A. C. (1995). The neural substrates underlying word generation: A bilingual functional-imaging study. Proceedings of the National Academy of Science, 92, 2899-2903.

Martin, A., Haxby, J. V., Lalonde, F. M., Wiggs, C. L., \& UngerLEIDER, L. G. (1995, October 6). Discrete cortical regions associated with knowledge of color and knowledge of action. Science, 270, 102-105.

Milner, B., Petrides, M., \& Smith, M. L. (1985). Frontal lobes and the temporal organization of memory. Human Neurobiology, 4 $137-142$

Moscovitch, M. (1982). Multiple dissociations of function in amnesia. In L. S. Cermak (Ed.), Human memory and amnesia (pp. 337 370). Hillsdale, NJ: Erlbaum.

Moscovitch, M. (1989). Confabulation and the frontal systems: Strategic versus associated retrieval in neuropsychological theories of memory. In H. L. Roediger III \& F. I. M. Craik (Eds.), Varieties of memory and consciousness: Essays in honour of Endel Tulving (pp. 133-155). Hillsdale, NJ: Erlbaum.

Nyberg, L., Cabeza, R., \& Tulving, E. (1996). PET studies of encoding and retrieval: The HERA model. Psychonomic Bulletin \& Review, 3, 135-148.
Petersen, S. E., Fox, P. T., Posner, M. I., Mintun, M. [A.], \& RaIChlE, M. E. (1988). Positron emission tomographic studies of the cortical anatomy of single-word processing. Nature, 331, 585-589.

Petersen, S. E., Fox, P. T., Posner, M. I., Mintun, M. [A.], \& RaICHLE, M. E. (1989). Positron emission tomographic studies of the processing of single words. Journal of Cognitive Neuroscience $1,153-170$

Petrides, M., Alivisatos, B., \& Evans, A. C. (1995). Functional activation of the human ventrolateral frontal cortex during mnemonic retrieval of verbal information. Proceedings of the National Acad emy of Science, 92, 5803-5807.

Petrides, M., Alivisatos, B., Evans, A. C., \& Meyer, E. (1993). Dissociation of human mid-dorsolateral from posterior dorsolateral frontal cortex in memory processing. Proceedings of the National Academy of Science, 90, 873-877.

Petrides, M., \& PANdya, D. N. (1994). Comparative architectonic analysis of the human and the macaque frontal cortex. In F. Boller \& J. Grafman (Eds.), Handbook of neuropsychology (Vol. 9, pp. 17 58). Amsterdam: Elsevier.

Posner, M. I., \& Raichle, M. E. (1994). Images of mind. New York: Scientific American Books.

Preuss, T. M., \& Goldman-RaKic, P. S. (1991). Myelo- and cytoarchitecture of the granular frontal cortex and surrounding regions in the strepsirhine Galago and the anthropoid primate Macaca. Journal of Comparative Neurology, 310, 429-474.

RaICHLE, M. E. (1987). Circulatory and metabolic correlates of brain function in normal humans. In F. Plum \& V. Mountcastle (Eds.), The handbook of physiology: Section 1. The nervous system: Vol. V. Higher functions of the brain: Pt. 1 (pp. 643-674). Bethesda, MD American Physiological Association.

Raichle, M. E., Fiez, J. A., Videen, T. O., MacLeod, A.-M. K. Pardo, J. V., Fox, P. T., \& Petersen, S. E. (1994). Practice-related changes in human brain functional anatomy during nonmotor learning. Cerebral Cortex, 4, 8-26.

RaJKowsKa, G., \& Goldman-Rakic, P. S. (1995a). Cytoarchitectonic definition of prefrontal areas in normal human cortex: I. Remapping of areas 9 and 46 using quantitative criteria. Cerebral Cortex, $5,307-322$.

RAJKowsKa, G., \& GoldMaN-RAKIC, P. S. (1995b). Cytoarchitectonic definition of prefrontal areas in normal human cortex: II. Variability in locations of areas 9 and 46 and relationship to the Talairach coordinate system. Cerebral Cortex, 5, 323-337.

SCHACTER, D. L. (1987). Memory, amnesia, and frontal lobe dysfunction. Psychobiology, 15, 21-36.

Schacter, D. L., Alpert, N. M., Savage, C. R., Rauch, S. L., \& AlBERT, M. S. (1996). Conscious recollection and the human hippocampal formation: Evidence from positron emission tomography. Proceedings of the National Academy of Science, 93, 321-325.

Schacter, D. L., \& Tulving, E. (EDS.) (1994). Memory systems 1994 Cambridge, MA: MIT Press.

Shimamura, A. P., Janowsky, J. S., \& SQuire, L. R. (1991). What is the role of frontal lobe damage in memory disorders? In H. Levin, H. M. Eisenberg, \& A. L. Benton (Eds.), Frontal lobe function and dysfunction (pp. 173-198). New York: Oxford University Press.

Squire, L. R., Ojemann, J. G., Miezin, F. M., Petersen, S. E., Videen, T. O., \& RAICHLE, M. E. (1992). Activation of the hippocampus in normal humans: A functional anatomical study of memory. Proceedings of the National Academy of Science, 89, 1837-1841.

Swick, D., \& KNIGHT, R. T. (in press). Is prefrontal cortex involved in cued recall? A neuropsychological test of PET findings. Neuropsychologia

Talairach, J., \& Tournoux, P. (1988). Co-planar stereotaxic atlas of the human brain. New York: Thieme Medical Publishers.

Tulving, E. (1983). Elements of episodic memory. New York: Oxford University Press

Tulving, E., Kapur, S., Craik, F. I. M., Moscovitch, M., \& Houle, S (1994). Hemispheric encoding/retrieval asymmetry in episodic memory: Positron emission tomography findings. Proceedings of the National Academy of Science, 91, 2016-2020.

Tulving, E., Kapur, S., Markowitsch, H. J., Craik, F. I. M., Habib, R., \& Houle, S. (1994). Neuroanatomical correlates of retrieval in 
episodic memory: Auditory sentence recognition. Proceedings of the National Academy of Science, 91, 2012-2015.

Wise, R., Chollet, F., Hadar, U., Friston, K. [J.], Hoffner, E., \& FRACKOWIAK, R. [S. J.] (1991). Distribution of cortical neural networks involved in word comprehension and word retrieval. Brain, 114, 1803-1817.

\section{NOTES}

1. The term resolution is most often used in neuroimaging to refer to how far apart two simultaneously imaged activations must be distanced before they can be identified as separate. I use the term here to refer to the absolute ability to resolve separate sources, which will likely (for PET) be better across multiple images than within single images (see Raichle, 1987, for an explanation). Furthermore, the currently available resolution of neuroimaging techniques is likely to improve as our methods advance. By the time this paper appears in press, studies may well have been done that demonstrate a higher level of resolution within the prefrontal cortex. In this regard, the values given for the ability of neuroimaging to resolve separate prefrontal activations should be considered an upper bound likely to improve as methods, especially those based on functional magnetic resonance imaging, are refined.

2. The issue of dissociating separate areas is difficult and should be approached carefully. Neuroimaging techniques often produce noisy data, and statistical tests are used to threshold data that are presented. For this reason, multiple areas might appear to be activated, owing to factors other than those related to functional anatomical boundaries. Dissociations across areas should be replicated and shown to be systematic across independent data sets before they are taken seriously

3. It is difficult to say exactly which Brodmann area an activation is located in, especially in the prefrontal cortex. This is because Brodmann's areas are defined by anatomical boundaries observed at the microscopic level. Gross anatomical landmarks cannot always be used to define the boundaries, because there is known between-subjects variability. For this reason, terminology such as "at or near Brodmann areas 44 or 45 " is used to reflect this uncertainty.

Rajkowska and Goldman-Rakic (1995a, 1995b) have recently mapped human variation in prefrontal areas based on cytoarchitectonic definitions. Importantly, the anatomic data from the individual subjects were placed into a standardized space identical to the space used by most neuroimaging groups. This extremely promising kind of analysis may eventually lead to a higher level of certainty about anatomic localization of activations.

4. The term semantic word retrieval is being used in its most general sense, referring to the generic access of words from long-term memory and not exclusively to access based on word meaning. The particular left inferior prefrontal area being discussed here appears to generalize across tasks that demand access to words based on meaning or based on other forms of cues that do not involve meaning (see Buckner, Raichle, et al., 1995; Klein et al., 1995). Some researchers may wish to subdivide these kinds of retrieval into semantic retrieval and lexical retrieval, respectively. Within this terminology, the left inferior prefrontal area is activated during both semantic and lexical retrieval
5. More areas than just the right anterior prefrontal cortex were additionally activated in the episodic version of the stem-completion task. These included several areas in the dorsal right prefrontal cortex and a less robust left prefrontal activation. An additional area in the medial parietal cortex (near precuneus) was also activated and has been reliably activated across many episodic memory retrieval tasks (e.g., Andreasen et al., 1995; Buckner, Petersen, et al., 1995; Buckner, Raichle, et al., 1995; Fletcher et al., 1995; Petrides et al., 1995).

6 . The idea of a brain area being used across many tasks to support retrieval of verbal information should not be taken to mean that the exact role of that area is well understood. Tentatively, it is suggested that the left inferior prefrontal area discussed in this review plays a role in representing verbal information while it is being retrieved. This idea is motivated, in part, by studies outside the domain of long-term memory which have shown activation of this area during working memory tasks (see Fiez et al., 1996, for a discussion of some of these findings).

The finding of overlapping brain areas being activated during verbal long-term memory retrieval and working memory illustrates an important point: processing events which we might separately call "working memory" and "long-term memory" may not be completely distinct at the functional anatomical level (see Buckner \& Tulving, 1995). Many forms of long-term memory retrieval likely involve a dynamic interaction between accessing information and the representation of that information. The representation and manipulation of information, often called working memory, is thus an intimate component of many kinds of long-term memory.

Some might argue that the overlap in activations between working memory and long-term memory suggests that brain areas involved in long-term memory have not truly been isolated. However, this assumes that there are brain pathways specialized only for long-term memory. It seems equally reasonable to assume that pathways important for long-term memory might map onto pathways being used for other kinds of memory function, with additional brain areas (or new interactions among brain areas) supporting demands specific to longterm memory. This should not be interpreted to mean that the areas more generally active are' not important for long-term memory. In the end, a complete description of all brain areas involved (and how they interact) will be necessary.

7. Another reason for examining multiple kinds of comparisons is to increase confidence in the data. Any single task image can contain noise or artifacts potentially producing spurious activations. By comparing multiple tasks with multiple control tasks, the likelihood that noise in any one task image will produce a false positive (Type I error) is reduced. Even in cases where a comparison seems redundant, more information can potentially be gained by examining that comparison-especially when thresholding procedures are used that only visualize activations reaching a certain magnitude (which is almost always the case).

(Manuscript received August 12, 1995; revision accepted for publication January $2,1996$. . 